\title{
Clinical presentation and management of uveal melanoma (Review)
}

\author{
ABELARDO RODRÍGUEZ ${ }^{1}$, ALFONSO DUEÑAS-GONZALEZ ${ }^{2}$ and SARAI DELGADO-PELAYO ${ }^{1}$ \\ ${ }^{1}$ Department of Pathology, Association for the Prevention of Blindness in Mexico I.A.P, Mexico City 04030; \\ ${ }^{2}$ Institute of Biomedical Research, UNAM/National Cancer Institute, Mexico City 14080, Mexico
}

Received July 8, 2015; Accepted July 25, 2016

DOI: $10.3892 / \operatorname{mco} .2016 .1037$

\begin{abstract}
Uveal melanoma (UM) is the most common primary intraocular malignancy in adults. The majority of the patients are Caucasian (97.8\%) and aged 50-80 years Choroidal melanoma is the predominant type $(86.3 \%)$. The clinical presentation may range from no symptoms over various types of visual disturbances to visual loss. Examination includes slit-lamp biomicroscopy, indirect ophthalmoscopy and diagnostic testing, such as B-scan ultrasonography. A number of patients with posterior UM are treated with plaque radiation therapy or enucleation. At present, targeted therapy includes inhibitors of the mitogen-activated protein kinase/mitogen-activated protein kinase kinase signaling pathway. UM disseminates hematogenously, with a high propensity for metastasis to the liver, which the most common site (93\% of the cases). While UM is uncommon, a significant proportion of affected patients succumb to this disease and new treatment options to improve patient survival are required.
\end{abstract}

\section{Contents}

1. Introduction

2. Clinical presentation and diagnosis

3. Molecular biology

4. Management

5. Metastasis

6. Prognosis

7. Conclusion

Correspondence to: Dr Sarai Delgado-Pelayo, Department of Pathology, Association for the Prevention of Blindness in Mexico I.A.P, 46 Vicente García Torres, Colonia Barrio San Lucas, Delegación Coyoacán, Mexico City 04030, Mexico

E-mail: saraimd88@gmail.com

Key words: uveal melanoma, ultrasonography, monosomy 3, mitogen-activated protein kinase/mitogen-activated protein kinase kinase, guanine nucleotide-binding protein $\mathrm{G}(\mathrm{q})$ subunit alpha, guanine nucleotide-binding protein subunit alpha-11

\section{Introduction}

Uveal melanoma (UM) is uncommon, accounting for only $3 \%$ of all melanoma cases. UM is the most common primary intraocular malignancy in adults, with an estimated incidence is 4-5 cases per million inhabitants/year in the United States and 5-7.4 cases in Europe $(1,2)$.

The majority of the affected patients are Caucasian (97.8\%). In addition to ethnicity, intrinsic host factors that appear to predispose to UM include light skin color, blonde hair and blue or light irides, dysplastic nevi, uveal nevi and oculodermal melanocytosis. Although cutaneous melanomas and UM share a common origin, i.e., the melanocyte, their clinical behavior and underlying molecular mechanisms differ significantly. Although exposure to sunlight has been suggested as a risk factor, its role appears to be complex. The majority of UM patients are aged 50-80 years, with a peak incidence in the 7 th decade of life and a mean age at diagnosis of 58 years. By contrast, melanoma of the iris is more common among young patients (aged $<20$ years). Compared with older patients, younger patients have a lower risk of metastatic disease $(3,4)$.

Any part of the uveal tract may be affected; however, choroidal melanoma is the predominant type, whereas melanomas of the iris and ciliary body are far less frequent. Choroidal and ciliary body melanomas are collectively referred to as posterior UM and differ from melanoma of the iris or anterior UM. Melanoma of the iris is the least common type of ocular melanoma and has a more benign clinical course compared with that of posterior UM (4).

We herein present a brief review of the current knowledge on UM, with emphasis on prognostic factors and recently discovered underlying molecular aberrations.

\section{Clinical presentation and diagnosis}

The clinical presentation of UM depends on the size and location of the tumor and may vary from asymptomatic, detected incidentally during eye examination, over various visual disturbances to visual loss. Loss of vision is usually caused by tumor involvement of the macula or by exudative retinal detachment. At diagnosis, the majority of UM patients are symptomatic; however, $\leq 30 \%$ may be asymptomatic (5).

The color, shape and number of lesions are important considerations in the diagnosis of UM, and differential diagnosis should include other conditions, such as retinal pigment epithelium (RPE) hyperplasia, RPE hypertrophy and melanocytoma. 
Choroidal melanoma usually presents as a dome- or mushroom-shaped subretinal mass. Ciliary body melanoma may be associated with a wide dilated pupil, presents as a dome-shaped or sessile lesion, and may cause lens displacement with consequent refractive and accommodation disturbances, localized cataract or increased intraocular pressure. Melanoma of the iris is usually asymptomatic and manifests as growth of a previously noted iris lesion or as a pigmented spot on the iris. Multifocal and bilateral lesions are more consistent with choroidal metastasis. Hemorrhage, inflammation and pain are rare, but may be seen in large tumors. An atypical presentation is diffuse melanoma, which is $<5 \mathrm{~mm}$ in thickness and encompasses more than a quarter of the uvea. Small lesions are difficult to diagnose $(4,6)$.

Examination of patients with suspected ocular melanoma includes slit-lamp biomicroscopy and diagnostic testing, such as B-scan ultrasonography, which is useful for characterizing and measuring the tumor. Ultrasound biomicroscopy allows for excellent visualization of tumors of the iris and ciliary body, and fluorescein angiography may also aid in the diagnosis (larger melanomas may exhibit an intrinsic tumor circulation referred to as double circulation). Although the majority of cases of UM may be accurately diagnosed non-invasively, fine-needle aspiration for cytological studies may be required, particularly if the result is likely to affect subsequent management. However, it has been reported that ocular tumors are missed or misdiagnosed in $23 \%$ of patients, resulting in more advanced stage at diagnosis and a higher rate of primary enucleation $(7,8)$.

\section{Molecular biology}

Advances in the understanding of the molecular mechanisms underlying UM have been made in recent years. Chromosomal alterations, such as monosomy 3 , which is associated with reduced survival, are present in $>80 \%$ of UM patients (9). In addition, gain of chromosome 8 and loss of chromosome 1 are associated with reduced survival. Loss of chromosome 3 as well as polysomy $8 \mathrm{q}$ are also associated with other poor prognostic factors (10).

Primary UM frequently harbours mutations resulting in constitutive activity of the mitogen-activated protein kinase (MAPK) pathway $(9,11)$.

At present, the gene most well-known to be associated with metastasis of UM is BAP1, which was identified by exome sequencing. The BAP1 gene encodes a de-ubiquitinating enzyme that binds to breast cancer 1, early onset (BRCA1) and BRCA1-associated RING domain protein 1 to form a heterodimeric complex with tumour suppressor function (12).

The MAPK kinase (MEK) inhibitor, selumetinib, improved progression-free and overall survival in UM patients when compared with temozolomide chemotherapy in a previous clinical trial (13).

Based on their gene expression profile, UM patients may be classified as class 1 (low risk of metastasis) or class 2 (high risk of metastasis), and this classification is associated with prognosis $(14,15)$.

Histone deacetylase inhibitors were reported to play a role in the treatment of patients with high-risk UM in an adjuvant setting prior to the emergence of overt metastatic disease (16). Approximately $2-3 \%$ of patients with UM may harbour a germline BAP1 mutation; identifying such patients is crucial (17).

$\mathrm{SF} 3 \mathrm{~B} 1$ is a component of the spliceosome and is implicated in the splicing of pre-mRNA. Its mutations are associated with a favorable prognosis in uveal melanoma; however, the underlying mechanisms have not been elucidated (18).

\section{Management}

The majority of patients with posterior UM are treated with enucleation. Other options include particle beam radiotherapy, transpupillary thermotherapy, laser photocoagulation, gamma knife stereotactic radiosurgery and local surgical resection. Management options range from observation to orbital exenteration, depending on the particular case (mainly tumor localization, size and local extension). Melanoma of the iris is commonly treated by surgical resection. Larger non-resectable tumors ( $>10$ apical height and $>16 \mathrm{~mm}$ diameter) may be treated by plaque radiotherapy or enucleation. Small (1.5-2.4 $\mathrm{mm}$ height and 5-16 mm diameter) and medium-sized (2.5-10 $\mathrm{mm}$ apical height and $\leq 16 \mathrm{~mm}$ diameter) choroidal tumors are mainly treated by radiation therapy, while large tumors, particularly if locally advanced, are mostly treated by enucleation or orbital exenteration. For large-sized tumors, neoadjuvant external radiation did not confer any advantage compared with enucleation alone. Small choroidal melanomas are initially managed by observation (4).

Novel insight into ocular melanoma biology has led to the investigation of immunotherapies, anti-angiogenic agents and targeted therapies, including kinase inhibitors such as sorafenib, sunitinib and imatinib (19).

Guanine nucleotide-binding protein $\mathrm{G}(\mathrm{q})$ subunit alpha/guanine nucleotide-binding protein subunit alpha-11 (GNAQ/GNA11) mutations, leading to aberrant activation of the MAPK pathway, render MEK in particular an attractive therapeutic target, with inhibitors of the MAPK/MEK signaling pathway. Falchook et al (20) did not observe any correlation between the mutational status of GNAQ/GNA11 and the clinical response to MEK inhibition; however, the analyzed exons were not specified in that study. Compared with temozolomide chemotherapy, the MEK inhibitor selumetinib prolonged progression-free survival in patients with melanoma (14).

\section{Metastasis}

UM disseminates hematogenously, with a high propensity for the liver, which is the most common metastatic site $(93 \%)$, followed by metastasis to the lung (24\%) and bones (16\%) (21). Metastases are the most common cause of UM-related mortality $(50 \%)$, which may occur despite successful local treatment. Metastases usually develop within the first 5 years following diagnosis (22).

UM was shown to be lethal in half of the affected patients, possibly due to clinically undetectable micrometastases already being present in the liver at the time of local therapy in class 2 patients or in those with monosomy 3 (23).

Surgical resection of liver metastases is beneficial in $<10 \%$ of patients with metastatic disease. Patients with 
metastasis exclusively to the liver and involvement of $<50 \%$ of the organ are eligible for this intervention. Chemotherapy is largely ineffective for metastatic UM, with response rates to dacarbazine or temozolomide of $<1 \%$ (24).

Monitoring by abdominal ultrasound examinations and serum liver function tests is required. Investigated tumor markers include $\mathrm{S}-100 \mathrm{~b}$ and tissue polypeptide-specific antigen (25).

To date, no chemotherapeutic, immunotherapeutic or targeted drug has achieved a reproducible overall response rate of $>10 \%$ in metastatic UM.

\section{Prognosis}

Prompt detection of metastasis allows for liver-directed management, such as chemoembolization; furthermore, molecular prognostic stratification allows high-risk patients to be enrolled in clinical trials of adjuvant therapies (3).

Mutational profiling may provide predictive information for determining the optimal therapeutic strategy. Pharmacological inhibitors of MEK, AKT and/or protein kinase $\mathrm{C}$ may be more effective in tumors with GNAQ11 mutations. Such data are obtained by direct sampling of primary or metastatic tumor tissue, or analysis of circulating tumor cells $(16,26,27)$.

Histopathological characteristics, including mitotic activity, epithelioid cell type, increased human leukocyte antigen expression, tumor infiltration by pro-angiogenic macrophages and lymphocytes, microvascular loops and certain extracellular matrix patterns are also predictors of poor prognosis (4).

\section{Conclusion}

While UM is uncommon, it is the cause of death in a considerable proportion of patients $(50 \%)$, and novel treatment options to improve patient survival are required. Knowledge of molecular aberrations in UM may provide new perspectives in the development of novel targeted therapeutic agents. This will hopefully improve the systemic treatment of patients with metastatic disease or prevent metastatic spread in those known to have tumors with high metastatic potential.

\section{References}

1. Singh AD, Turell ME and Topham AK: Uveal melanoma: Trends in incidence, treatment and survival. Ophthalmology 118: 1881-1885, 2011.

2. Vicente MA, Saornil C, García-Álvarez C, Almaraz A, Alonso Martínez P, Frutos-Baraja JM and López-Lara F: Uveal melanoma: Clinical characteristics, treatment and survival in a series of 500 patients. Arch Soc Esp Oftalmol 88: 433-438, 2013.

3. Schoenfield L: Uveal melanoma: A pathologist's perspective and review of translational developments. Adv Anat Pathol 21: 138-143, 2014.

4. Jovanovic P, Mihajlovic M, Djordjevic-Jocic J, Vlajkovic S, Cekic S and Stefanovic V: Ocular melanoma: An overview of the current status. Int J Clin Exp Pathol 6: 1230-1244, 2013.

5. Eskelin S and Kivelä T: Mode of presentation and time to treatment of uveal melanoma in Finland. Br J Ophthalmol 86 333-338, 2002.
6. Shields CL, Cater J, Shields JA, Singh AD, Santos MC and Carvalho C: Combination of clinical factors predictive of growth of small choroidal melanocytic tumors. Arch Ophthalmol 118: 360-364, 2000

7. Damato EM and Damato BE: Detection and time to treatment of uveal melanoma in the United Kingdom: An evaluation of 2,384 patients. Ophthalmology 119: 1582-1589, 2012.

8. Harbour JW, Augsburger JJ and Char DH: Gene expression profiling versus TNM classification. Ophthalmology 120: e52-e 53, 2013.

9. Sapna P: Latest developments in the biology and management of uveal melanoma. Curr Oncol Rep 15: 509-516, 2013.

10. Damato B, Dopierala JA and Coupland SE: Genotypic profiling of 452 choroidal melanomas with multiplex ligation dependent probe amplification. Clin Cancer Res 16: 6083-6092, 2010.

11. Van Raamsdonk CD, Griewank KG, Crosby MB, Garrido MC, Vemula S, Wiesner T, Obenauf AC, Wackernagel W, Green G, Bouvier N, et al: Mutations in GNA11 in uveal melanoma. N Engl J Med 363: 2191-2199, 2010.

12. Matthew G, Field J and Harbour W: Recent developments in prognostic and predictive testing in uveal melanoma. Curr Opin Ophthalmol 25: 234-239, 2014.

13. Selumetinib shows promise in metastatic uveal melanoma. Cancer Discov 3: OF8, 2013.

14. Onken MD, Worley LA, Ehlers JP and Harbour JW: Gene expression profiling in uveal melanoma reveals two molecular classes and predicts metastatic death. Cancer Res 64: 7205-7209, 2004.

15. Harbour JW, Onken MD, Roberson ED, et al: Frequent mutation of BAP1 in metastasizing uveal melanomas. Science 330: 1410-1413, 2010.

16. Landreville S, Agapova OA, Matatall KA, Kneass ZT, Onken MD, Lee RS, Bowcock AM and Harbour JW: Histone deacetylase inhibitors induce growth arrest and differentiation in uveal melanoma. Clin Cancer Res 18: 408-416, 2012.

17. Carbone M, Yang H, Pass HI, Krausz T, Testa JR and Gaudino G: BAP1 and cancer. Nat Rev Cancer 13: 153-159, 2013.

18. Harbour JW, Roberson ED, Anbunathan H, Onken MD, Worley LA and Bowcock AM: Recurrent mutations at codon 625 of the splicing factor SF3B1 in uveal melanoma. Nat Genet 45: 133-135, 2013.

19. Buder K, Gesierich A, Gelbrich G and Goebeler M: Systemic treatment of metastatic uveal melanoma: Review of literature and future perspectives. Cancer Medicine 2: 674-686, 2013.

20. Falchook GS, Lewis KD, Infante JR, Gordon MS, Vogelzang NJ, DeMarini DJ, et al: Activity of the oral MEK inhibitor trametinib in patients with advanced melanoma: a phase 1 dose-escalation trial. Lancet Oncol 13: 782-789, 2012.

21. Kujala E, Mäkitie T and Kivelä T: Very long-term prognosis of patients with malignant uveal melanoma. Invest Ophthalmol Vis Sci 44: 4651-4659, 2003.

22. Buzzacco DM, Abdel-Rahman MH, Park S, Davidorf F, Olencki T and Cebulla CM: Long-term survivors with metastatic uveal melanoma. Open Ophthalmol J 6: 49-53, 2012.

23. Hendler K, Pe'er J, Kaiserman I, Baruch R, Kalickman I, Barak V and Frenkel S: Trends in liver function tests: A comparison with serum tumor markers in metastatic uveal melanoma (part 2). Anticancer Res 31: 351-358, 2011.

24. Patel SP: Latest developments in the biology and management of uveal melanoma. Curr Oncol Rep 15: 509-516, 2013.

25. Harbour JW: A prognostic test to predict the risk of metastasis in uveal melanoma based on a 15-gene expression profile. Methods Mol Biol 1102: 427-440, 2014.

26. Chen X, Wu Q, Tan L, Porter D, Porter D, Jager MJ, Emery C and Bastian BC: Combined PKC and MEK inhibition in uveal melanoma with GNAQ and GNA11 mutations. Oncogene 33: 4724-4734, 2014.

27. Ambrosini G, Musi E, Ho AL, de Stanchina E and Schwartz GK: Inhibition of mutant GNAQ signaling in uveal melanoma induces AMPK-dependent autophagic cell death. Mol Cancer Ther 12: 768-776, 2013. 